\title{
Fabrication and Characterization of Prussian Blue-Derived Iron Carbide-Iron Oxide Hybrid on Reduced Graphene Oxide Nanosheets ${ }^{\dagger}$
}

\author{
Alowasheeir Azhar ${ }^{1 \ddagger}$, Jacob Earnshaw ${ }^{2 \ddagger}$, Mohamed Barakat Zakaria ${ }^{1,2 *}$, Ping Cheng ${ }^{2}$, \\ Yusuf Valentino Kaneti ${ }^{1}$, Md. Shahriar A. Hossain ${ }^{2,3}$, Saad M. Alshehri ${ }^{4}$, \\ Tansir Ahamad ${ }^{4}$, Yusuke Yamauchi ${ }^{2,5,6}$ and Jongbeom Na ${ }^{1,2 *}$ \\ ${ }^{1}$ International Center for Materials Nanoarchitectonics (MANA), National Institute for Materials Science (NIMS), Japan \\ ${ }^{2}$ Australian Institute for Bioengineering and Nanotechnology (AIBN), The University of Queensland, Australia \\ ${ }^{3}$ School of Mechanical \& Mining Engineering, Faculty of Engineering, Architecture and Information Technology (EAIT), The University of \\ Queensland, Australia \\ ${ }^{4}$ Department of Chemistry, College of Science, King Saud University, Saudi Arabia \\ ${ }^{5}$ School of Chemical Engineering, The University of Queensland, Australia \\ ${ }^{6}$ Department of Plant \& Environmental New Resources, Kyung Hee University, South Korea
}

\begin{abstract}
This work demonstrates the fabrication of a nanoporous iron carbide-iron oxide/reduced graphene oxide (IC-IO/ rGO) hybrid via a controlled one-step thermal treatment of Prussian blue (PB)/GO hybrid at $450{ }^{\circ} \mathrm{C}$ under $\mathrm{N}_{2}$ flow. The $\mathrm{PB} / \mathrm{GO}$ hybrid is initially prepared through the in-situ deposition of $\mathrm{PB}$ nanoparticles on the GO sheets through electrostatic interactions. The morphological analysis of the hybrid reveals the uniform coverage of the rGO sheets by IC-IO nanoparticles and the even distribution of carbon $(\mathrm{C})$, oxygen $(\mathrm{O})$, and iron $(\mathrm{Fe})$ on the rGO nanosheets. As a result of the hybrid composition and controlled morphology, the surface area of the obtained IC-IO/rGO hybrid $\left(\sim 40 \mathrm{~m}^{2} / \mathrm{g}\right.$ ) is significantly enhanced compared to those of the calcined GO sheets and PB nanoparticles (without $\mathrm{GO})$.
\end{abstract}

Keywords: metal-organic frameworks, porous coordination polymers, metal oxides, metal carbides, graphene oxide

\section{Introduction}

Metal-organic frameworks (MOFs) or porous coordination polymers (PCPs) have attracted significant interest as porous materials because of their high surface area, large pore volume, controllable composition and pore size, etc. (Wang Z.-L. et al., 2018; Salunkhe R. R. et al., 2016; Kaneti Y. V. et al., 2017). Prussian blue (PB) and its analogues (PBAs) exhibit several attractive characteristics, such as open framework structures, high thermal stability, and good redox activity. These CPs have been employed in numerous applications, including drug delivery (Lin W. et al., 2009), energy storage (Paolella A. et al., 2017), sep-

\footnotetext{
Received 27 May 2020; Accepted 21 July 2020

J-STAGE Advance published online 29 August 2020

Authors with equal contribution

1-1 Namiki, Tsukuba, Ibaraki 305-0044, Japan

Brisbane, Queensland 4072, Australia

* Corresponding authors: Mohamed Barakat Zakaria and Jongbeom $\mathrm{Na}$;

E-mail:m.barakat@uq.edu.au;j.na@uq.edu.au
}

aration (Bureekaew S. et al., 2008), and so on (Doty F. et al., 2009; Ishizaki M. et al., 2013). Nevertheless, the poor conductivity and low chemical stability of PB presents major challenges for their practical applications (Salunkhe R. R. et al., 2015). Therefore, extensive efforts are needed to enhance the stability and conductivity of PB and its derived materials. To date, $\mathrm{PB}$ and $\mathrm{PBAs}$ have been employed as novel precursors for obtaining various transition metal compounds, including metal oxides and metal carbides (Azhar A. et al., 2019a). Generally, the calcination of PB in air produces iron oxide (IO) as a result of the removal of the cyano-group and the oxidation of the iron species. Our group previously synthesized $\mathrm{PB}$ and completely transformed it into nanoporous iron oxide hybrids (Azhar A. et al., 2019b), such as $\beta-\mathrm{Fe}_{2} \mathrm{O}_{3}$ (Machala L. et al., 2013), and mixed crystalline phases of iron oxide ( $\alpha, \gamma$ and $\beta$-phases) (Roy X. et al., 2011). On the contrary, when pure PB was heated under inert atmosphere, the formation of metallic iron and iron carbide (IC) $\left(\mathrm{Fe}_{7} \mathrm{C}_{3}, \mathrm{Fe}_{2} \mathrm{C}\right.$, and $\left.\mathrm{Fe}_{3} \mathrm{C}\right)$ was observed (Zakaria M. B. et al., 2016). Very recently, pure nickel carbide $\left(\mathrm{Ni}_{3} \mathrm{C}\right)$ was successfully prepared from 
gyang $\$$ bridged CPs through a thermal treatment under inert aturosphere at $450{ }^{\circ} \mathrm{C}$ (Zakaria M. B. et al., 2019). Despite some progress, the construction of PB-derived metal carbide/carbon hybrids are still rarely reported.

Recently, carbonaceous materials, such as graphite, graphene oxide (GO), reduced graphene oxide (rGO), and carbon nanotubes (CNTs) have been widely used to enhance the functional performance of PB-based materials (Daneshvar F. et al., 2018; Azhar A. et al., 2018). The oxygen-containing functional groups on the surface of the two-dimensional (2D) GO nanosheets can serve as excellent sites for the in-situ growth of PB nanostructures (Lee T. et al., 2015). Our group has demonstrated the fabrication of several cyano-bridged CPs grown on GO nanosheets by a variety of methods (Zakaria M. B. et al., 2019). Herein, we report the synthesis of a hybrid material combining GO sheets with $\mathrm{PB}$ nanoparticles which can be subsequently transformed into a nanoporous $\mathrm{IC}-\mathrm{IO} / \mathrm{rGO}$ hybrid under nitrogen atmosphere at $450{ }^{\circ} \mathrm{C}$. The obtained IC-IO/rGO hybrid has been thoroughly characterized in terms of its composition, morphology, thermal decomposition behavior, and textural properties.

\section{Experimental}

\subsection{Chemicals}

Sodium ferrocyanide(II) decahydrate $\left(\mathrm{Na}_{4}\left[\mathrm{Fe}(\mathrm{CN})_{6}\right] \cdot 10 \mathrm{H}_{2} \mathrm{O}, \geq 99 \%\right)$ was purchased from Sigma-Aldrich (Japan). The sulfuric acid solution $\left(\mathrm{H}_{2} \mathrm{SO}_{4}\right.$, $98 \%$ ) was obtained from Nacalai Tesque (Japan). Potassium hydroxide $(\mathrm{KOH})$, sodium nitrate $\left(\mathrm{NaNO}_{3}, \geq 99 \%\right)$, and ferric chloride hexahydrate $\left(\mathrm{FeCl}_{3} \cdot 6 \mathrm{H}_{2} \mathrm{O}, \geq 98 \%\right)$ were sourced from FUJIFILM Wako Corporation (Japan). Graphite nanoplatelets (N008-100-N, thickness 100 nm) were obtained from Angstron materials (USA). Potassium permanganate $\left(\mathrm{KMnO}_{4}, \geq 99 \%\right)$ and hydrogen peroxide solution $\left(\mathrm{H}_{2} \mathrm{O}_{2}, 30\right.$ wt.\% in $\left.\mathrm{H}_{2} \mathrm{O}\right)$ were purchased from Kanto Chemicals (Japan). All chemicals were utilized as received without further purification.

\subsection{Preparation of GO nanosheets}

The thin GO nanosheets were fabricated by employing the modified Hummer's approach (Tanaka S. et al., 2017). In the first step, $0.33 \mathrm{~g}$ of graphite powder and $0.17 \mathrm{~g}$ of $\mathrm{NaNO}_{3}$ were mixed together and stirred. Following this, $7.67 \mathrm{~mL}$ of concentrated $\mathrm{H}_{2} \mathrm{SO}_{4}$ solution was slowly poured into this suspension and then stirred for $1 \mathrm{~h}$. Next, $1.0 \mathrm{~g}$ of $\mathrm{KMnO}_{4}$ was added into the mixture solution which was placed in an ice bath below $20^{\circ} \mathrm{C}$. The mixture was subsequently stirred at $35^{\circ} \mathrm{C}$ for $2 \mathrm{~h}$ and distilled water $(83 \mathrm{~mL})$ was added into this mixture solution under strong stirring. After that, $1.67 \mathrm{~mL}$ of aqueous $\mathrm{H}_{2} \mathrm{O}_{2}$ solution $(30 \% \mathrm{w} / \mathrm{w})$ was added into the suspension. The final GO suspension was washed a number of times with a diluted $\mathrm{HCl}$ solution and distilled water. Finally, this GO suspension was sonicated in distilled water to exfoliate the GO sheets. The GO sheets were collected by centrifugation and subjected to repeated washing with distilled water, before being dried at ambient temperature, followed by a final drying in vacuum at $60{ }^{\circ} \mathrm{C}$ overnight. The as-prepared GO powder was dispersed in water to prepare an aqueous $\mathrm{GO}$ solution $\left(2 \mathrm{mg} \mathrm{mL}^{-1}\right)$ to be used in subsequent steps.

\subsection{In-situ deposition of PB nanoparticles on GO nanosheets (PB/GO hybrid) and conversion to iron carbide-iron oxide/rGO hybrid}

Typically, $40 \mathrm{~mL}$ of $0.299 \mathrm{mM} \mathrm{FeCl} \cdot 6 \mathrm{H}_{2} \mathrm{O}$ solution was poured into the GO solution $\left(20 \mathrm{~mL}, 2 \mathrm{mg} \mathrm{mL}^{-1}\right)$ and stirred for $0.5 \mathrm{~h}$. The mixture solution was slowly mixed with $40 \mathrm{~mL}$ of $\mathrm{Na}_{4}\left[\mathrm{Fe}(\mathrm{CN})_{6}\right] \cdot 10 \mathrm{H}_{2} \mathrm{O}$ solution $(0.358 \mathrm{mM})$ and stirred for further $0.5 \mathrm{~h}$, before being aged for two days. The product was isolated via centrifugation and washed multiple times with water and ethanol, and finally dried at room temperature. The porous IC-IO/rGO hybrid was achieved by calcining the $\mathrm{PB} / \mathrm{GO}$ hybrid at $450^{\circ} \mathrm{C}$ for an hour under nitrogen $\left(\mathrm{N}_{2}\right)$ flow with a ramping rate of $5{ }^{\circ} \mathrm{C} \mathrm{min}{ }^{-1}$. For comparison, pristine $\mathrm{PB}$ nanoparticles without $\mathrm{GO}$ sheets were also prepared and heated under the same conditions.

\subsection{Characterization}

The purity and compositions of the samples were checked by X-ray diffraction (XRD) with a Rigaku RINT 2500X diffractometer utilizing $\mathrm{Cu} \mathrm{K \alpha}(1.5406 \AA)$ radiation. Nitrogen sorption isotherms were collected using a Quantachrome Autosorb at $77 \mathrm{~K}$. To dehydrate the samples, they were subjected to degassing at $250{ }^{\circ} \mathrm{C}$ for $16 \mathrm{~h}$ prior to the BET measurement. Morphological observations of the products were conducted using both scanning electron microscope (SEM, Hitachi SU8000) and transmission electron microscope (TEM, JEOL JEM2100F). The infrared (IR) spectra of the samples were collected using a Thermoscientific Nicolet 4700 spectrometer (Waltham, MA, USA). Raman spectroscopy measurements were performed using a Horiba-Jovin Yvon T64000 Raman spectrometer. Thermogravimetry (TG) measurements were carried out with a Hitachi HT-Seiko Instrument Exter 6300 under $\mathrm{N}_{2}$ atmosphere from 30 to $550^{\circ} \mathrm{C}$ at a ramping rate of $5^{\circ} \mathrm{C} \mathrm{min}^{-1}$. 

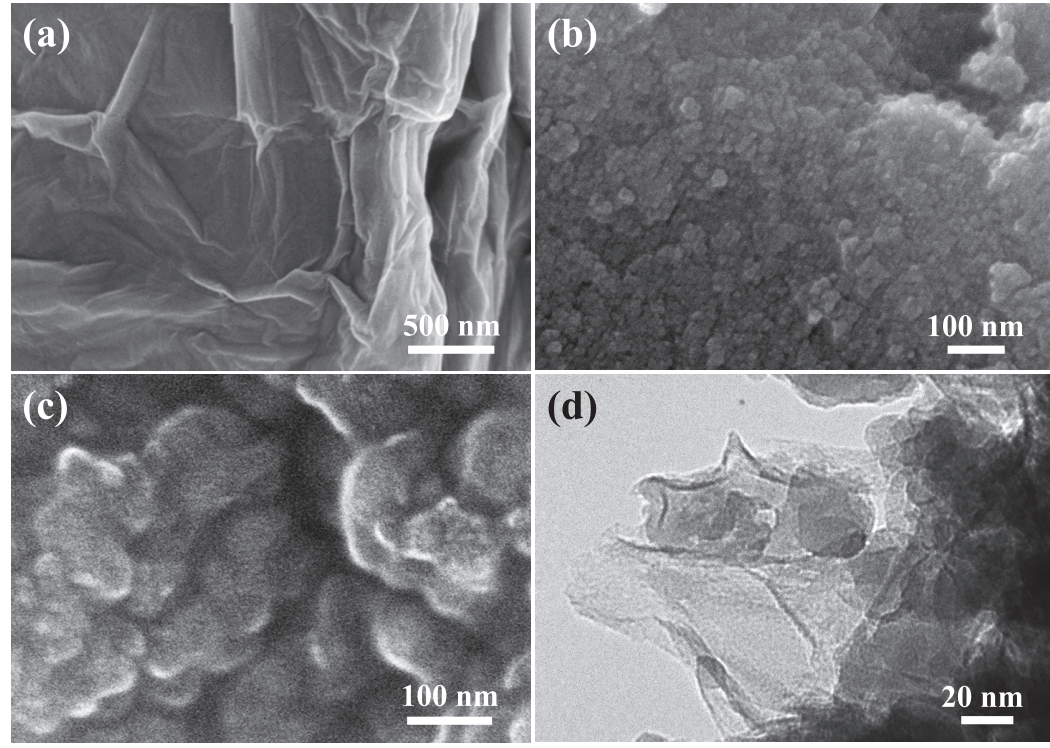

(d)

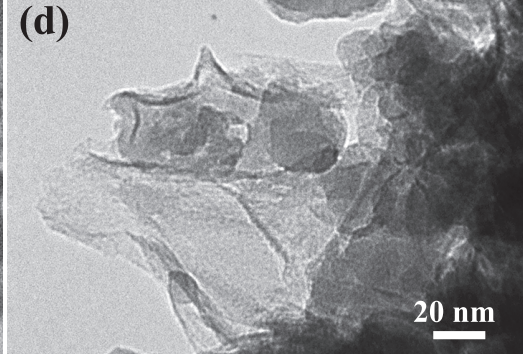

Fig. 1 (a, b) SEM images of (a) GO nanosheets and (b) PB nanoparticles. (c) SEM and (d) TEM images of the as-synthesized PB/GO hybrid

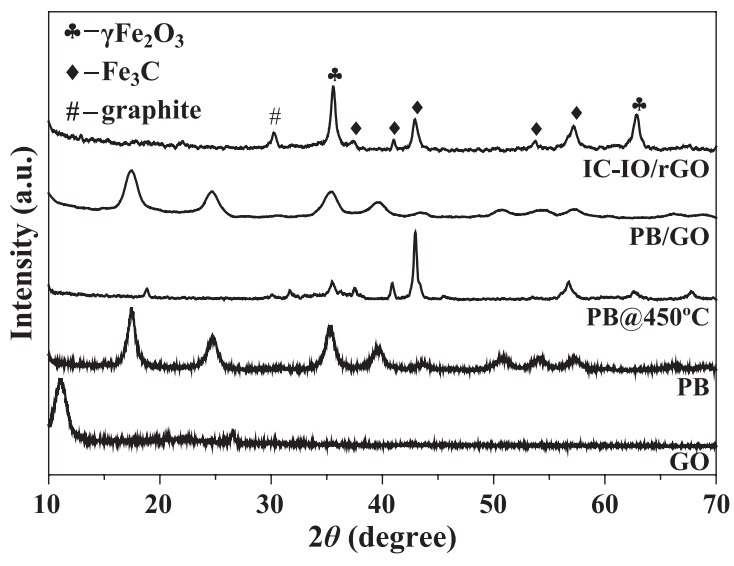

Fig. 2 Wide-angle XRD patterns of GO, PB, PB/GO hybrid and IC-IO/ rGO hybrid. For comparison, the XRD pattern of calcined PB without GO is also shown.

\section{Results and discussion}

The $\mathrm{PB} / \mathrm{GO}$ hybrid was obtained by the in-situ deposition of PB nanoparticles on the GO sheets (Azhar A. et al., 2019b). First, the GO sheets were synthesized by the exfoliation of graphite based on the modified Hummer's method (Tanaka S. et al., 2017). The SEM image of the prepared GO sheets (Fig. 1a) shows a two-dimensional (2D) crumpled sheet-like structure. The zeta potential measurements reveal the change in the surface charge of the GO sheets from negative to positive charge after the modification with PB nanoparticles. The morphology of the PB/the GO hybrid is depicted in Fig. 1c, which shows the wrapping of the PB nanoparticles by the GO sheets (i.e., the surface of the GO sheets is bumpy.). The TEM image further shows that the PB nanoparticles are successfully deposited on the surface of the GO sheets
(Fig. 1d). For comparison, pristine PB without GO sheets was also prepared (Fig. 1b). Fig. 2 shows the XRD pattern of the $\mathrm{PB} / \mathrm{GO}$ hybrid which reveals the diffraction peaks belonging to face-centered cubic (fcc) phase of PB (JCPDS No. 73-0687) (Cao L. et al., 2010). Importantly, after the modification with $\mathrm{PB}$, the primary diffraction peak belonging to the GO nanosheets disappears, possibly due to the nearly complete surface coverage of the GO sheets by the PB nanoparticles (Islam M. N. et al., 2018).

Further compositional analyses of the PB nanoparticles, GO sheets, and PB/GO hybrid were carried out using fourier-transform infrared (FTIR) spectroscopy. The FTIR spectrum of the PB/GO hybrid (Fig. 3a) shows the presence of strong bands belonging to the cyano $(\mathrm{CN})$ group of $\mathrm{PB}\left(\mathrm{Fe}^{2+}-\mathrm{CN}-\mathrm{Fe}^{3+}\right)$ (Ge C.-X. et al., 2018). The peaks originating from oxygen-containing functional groups (Vermisoglou E. et al., 2014) on the GO surface is significantly reduced following the modification with PB nanoparticles, indicating the complete reduction of GO to reduced graphene oxide (rGO) (Ren S. et al., 2012) and the formation of $\mathrm{PB}$ on $\mathrm{GO}$ sheets.

The thermal degradation of $\mathrm{PB}$ under nitrogen flow was studied by TGA (Fig. 4). The TG curve of pure PB shows multi-step weight loss (Sun D. et al., 2019). In the first stage, lattice water and adsorbed water molecules in the structure are removed at temperatures between 30 and $210^{\circ} \mathrm{C}$. The second weight loss $\left(210-420^{\circ} \mathrm{C}\right)$ can be attributed to the loss of the cyano $(\mathrm{CN})$ group present in the PB nanoparticles, and the final weight loss above $420{ }^{\circ} \mathrm{C}$ is correlated to the formation of IC. The thermal stability of the pure GO nanosheets under inert atmosphere was also investigated by TG measurements (Fig. 4). The evaporation of adsorbed water molecules and the removal of labile oxygen functional groups occur at $\sim 250{ }^{\circ} \mathrm{C}$ and no further 

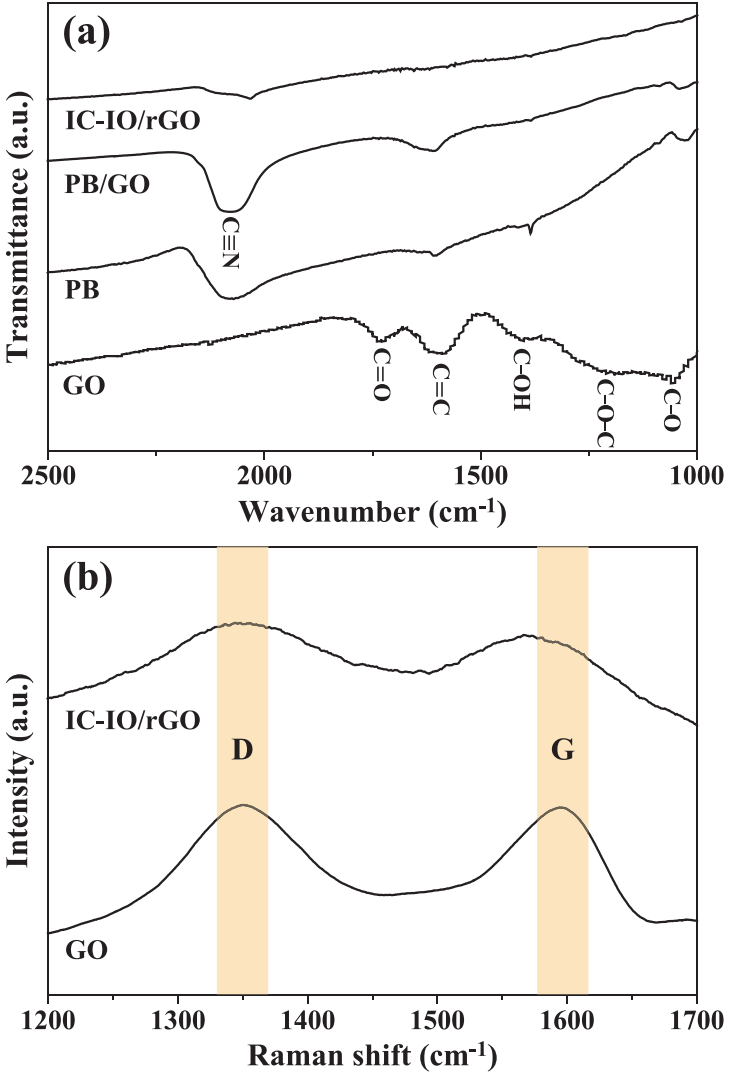

Fig. 3 (a) FTIR spectra of GO, PB, PB/GO hybrid, and IC-IO/rGO hybrid. (b) Raman spectra of GO and IC-IO/rGO.

weight loss is observed (Park S. et al., 2011).

As depicted in Scheme 1, the thermal treatment of the $\mathrm{PB} / \mathrm{GO}$ hybrid at $450{ }^{\circ} \mathrm{C}$ under $\mathrm{N}_{2}$ atmosphere results in the formation of the IC-IO/rGO hybrid. The crystal structure of this hybrid after calcination was examined by wide-angle XRD (Fig. 2). Some peaks are assignable to iron carbide $\left(\mathrm{Fe}_{3} \mathrm{C}, \mathrm{IC}\right)$ (Fletcher D. et al., 2019), while the other peaks at $35.5^{\circ}$ and $62.9^{\circ}$ can be attributed to iron oxide $\left(\gamma-\mathrm{Fe}_{2} \mathrm{O}_{3}\right)$ (Zhu K. et al., 2018), suggesting the presence of multiple iron phases after the heat treatment. Compared to the calcined $\mathrm{PB}$ (without GO), the relative intensity of the IO peak in the IC-IO/rGO hybrid is increased, which may be due to the reaction of iron species with oxygen functional groups of GO sheets.

Fig. 5a displays the SEM image of the IC-IO/rGO hy-

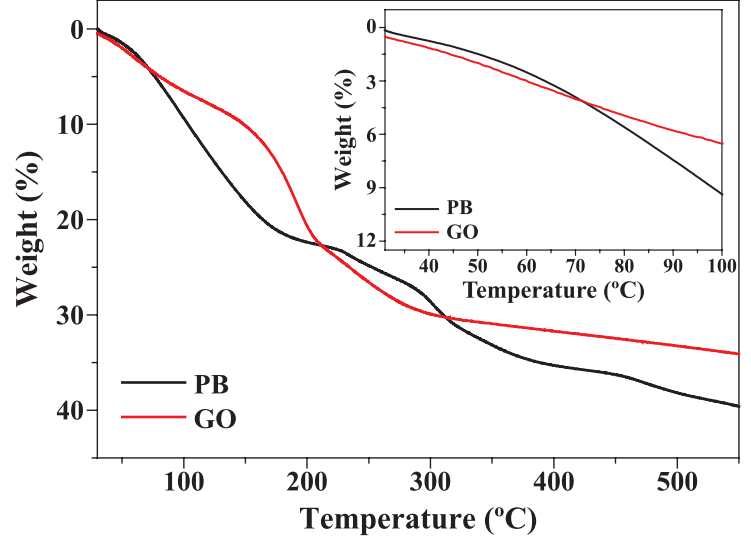

Fig. 4 TGA data of (a) PB and (b) GO. The measurement was carried out in $\mathrm{N}_{2}$ atmosphere.

brid, which is composed of irregular and porous structures due to the removal of the organic group after calcination. Also, some IC-IO hybrid nanoparticles can be observed on the surface of the GO nanosheets. The TEM image clearly reveals the uniform decoration of the rGO sheets by IC-IO nanoparticles (Fig. 5b). The corresponding elemental mapping images in Fig. $\mathbf{5 c}-\mathbf{f}$ confirm the uniform distribution of carbon (C), oxygen (O), and iron $(\mathrm{Fe})$ on the rGO nanosheets.

The $\mathrm{N}_{2}$ sorption isotherms of the calcined $\mathrm{PB}$ and the IC-IO/rGO hybrid are given in Fig. 6. The specific surface area of the IC-IO/rGO hybrid $\left(38.7 \mathrm{~m}^{2} / \mathrm{g}\right)$ is significantly larger than those of the calcined GO sheets $\left(1.25 \mathrm{~m}^{2} / \mathrm{g}\right)$ (Zakaria M. B. et al., 2019) and calcined PB $\left(18.2 \mathrm{~m}^{2} / \mathrm{g}\right)$. This is likely due to the presence of IC-IO nanoparticles which may serve as effective spacers between the GO sheets, thus preventing the stacking of the GO sheets during the thermal treatment.

The FTIR spectrum of the IC-IO/rGO hybrid (Fig. 3a) clearly shows the disappearance of many oxygencontaining functional groups, which suggests the successful reduction of $\mathrm{GO}$ sheets to reduced $\mathrm{GO}(\mathrm{rGO})$ ( $\mathrm{Liu} \mathrm{H}$. et al., 2015). Additionally, a weak IR band belonging to the $\mathrm{CN}$ group is also observed. Raman spectra of the GO sheets before and after the thermal treatment are compared in Fig. 3b. The $D$ and $G$ bands are clearly observed. However, the positions of the $D$ and $G$ bands are shifted after the

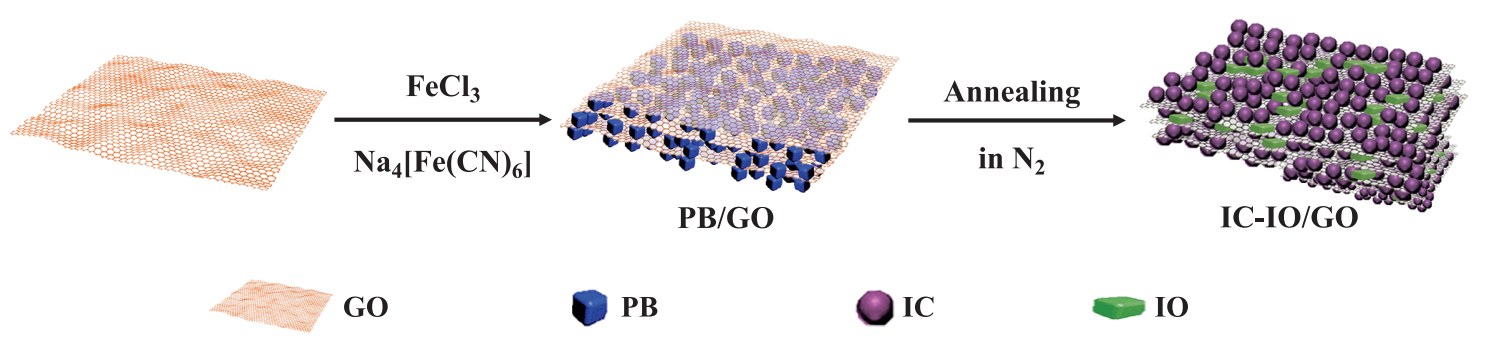

Scheme 1 Schematic illustration of the in-situ growth of PB nanoparticles on GO sheets (PB/GO hybrid) and the subsequent thermal decomposition to IC-IO/rGO hybrid. 

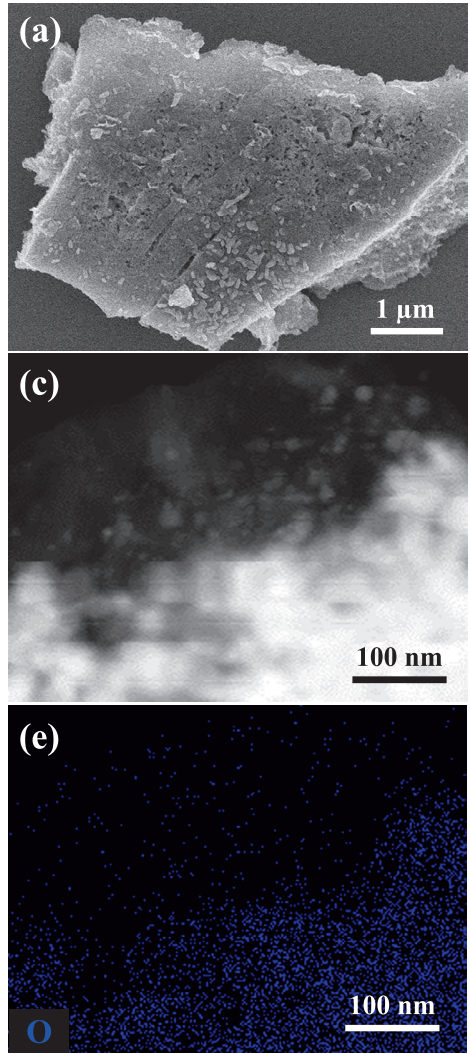

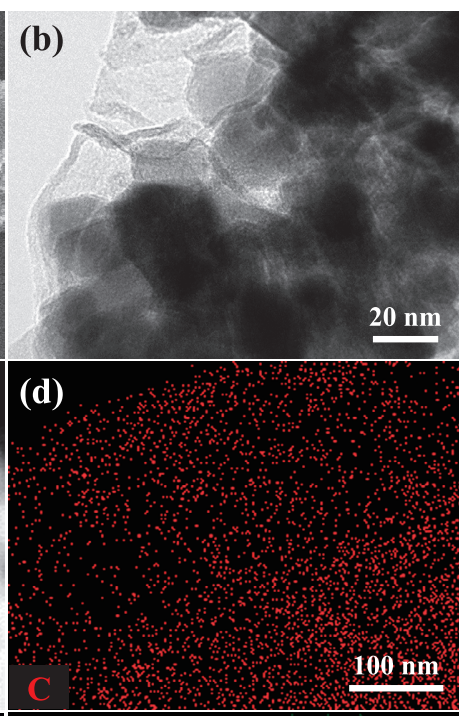

(f)

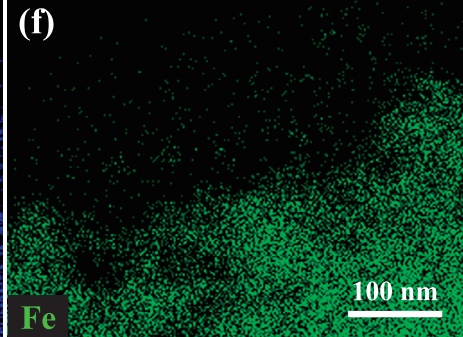

Fig. 5 (a) SEM and (b) TEM images of the IC-IO/rGO hybrid obtained by the thermal treatment of the $\mathrm{PB} / \mathrm{GO}$ hybrid at $450{ }^{\circ} \mathrm{C}$ for $1 \mathrm{~h}$ under $\mathrm{N}_{2}$ atmosphere. (c) HAADF-STEM image of the IC-IO/rGO hybrid and (d-f) the corresponding elemental mapping for (d) carbon, (e) oxygen, and (f) iron.

(a)
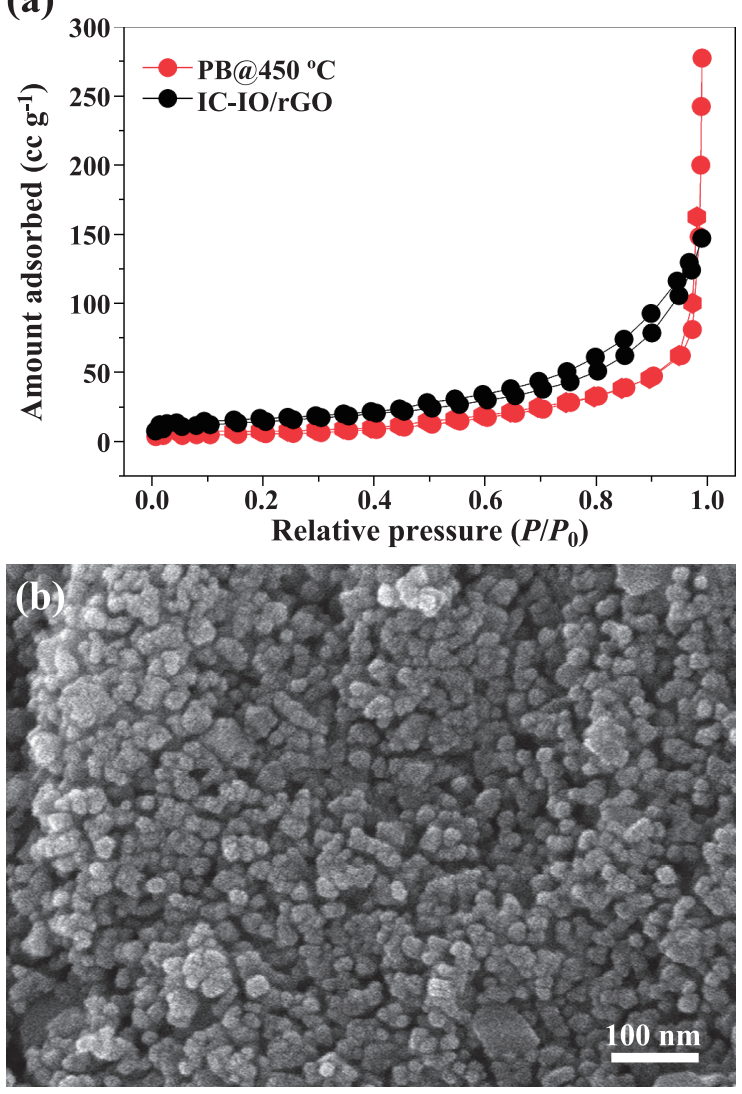

Fig. 6 (a) $\mathrm{N}_{2}$ adsorption-desorption isotherms of $\mathrm{PB}$ calcined at $450{ }^{\circ} \mathrm{C}$ and the IC-IO/rGO hybrid. (b) SEM image of calcined-PB nanoparticles. thermal treatment. Compared to the $I_{\mathrm{D}} / I_{\mathrm{G}}$ value of pure GO (1.02), the $I_{\mathrm{D}} / I_{\mathrm{G}}$ value of the IC-IO/rGO hybrid is higher (1.12), suggesting the increase of structural defects in the GO sheets after calcination.

\section{Conclusions}

In summary, we have successfully achieved the in-situ deposition of PB nanoparticles on the surface of GO sheets through the interaction of $\mathrm{PB}$ with the oxygen-containing functional groups of $\mathrm{GO}$ nanosheets. This $\mathrm{PB} / \mathrm{GO}$ hybrid can be converted to the IC-IO/rGO hybrid via a onestep thermal treatment at $450{ }^{\circ} \mathrm{C}$ under $\mathrm{N}_{2}$ flow with well-retained morphology. This finding indicates that the presence of rGO during thermal treatment helps to promote the formation of iron carbide at high temperatures. The surface area of the obtained IC-IO/rGO hybrid $\left(\sim 40 \mathrm{~m}^{2} / \mathrm{g}\right)$ is superior to those of the calcined GO sheets and the calcined $\mathrm{PB}$ without GO. This hybrid material composed of multiple compositions may have many potential applications in supercapacitors and oxygen reduction reaction (ORR).

\section{Acknowledgements}

This work was conducted at the Queensland node of 
thoe Atrstralian National Fabrication Facility (ANFF-Q), a ompany established under the National Collaborative Research Infrastructure Strategy to provide nano and microfabrication facilities for Australian researchers. S.M.A., T.A., and Y.Y. thank Researchers Supporting Project Number (RSP-2020/6), King Saud University, Riyadh, Saudi Arabia.

\section{References}

Azhar A., Li Y., Cai Z., Zakaria M.B., Masud M.K., Hossain M.S.A., Kim J., Zhang W., Na J., Yamauchi Y., Hu M., Nanoarchitectonics: a new materials horizon for Prussian blue and its analogues, Bulletin of the Chemical Society of Japan, 92 (2019a) 875-904. DOI: 10.1246/bcsj.20180368

Azhar A., Yamauchi Y., Allah A.E., Alothman Z.A., Badjah A.Y., Naushad M., Habila M., Wabaidur S., Wang J., Zakaria M.B., Nanoporous iron oxide/carbon composites through in-situ deposition of Prussian blue nanoparticles on graphene oxide nanosheets and subsequent thermal treatment for supercapacitor applications, Nanomaterials, 9 (2019b) 776. DOI: 10.3390/nano9050776

Azhar A., Zakaria M.B., Lin J., Chikyow T., Martin D.J., Alghamdi Y.G., Alshehri A.A., Bando Y., Hossain M.S.A., Wu K.C.-W., Kumar N.A., Yamauchi Y., Graphene-wrapped nanoporous nickel-cobalt oxide flakes for electrochemical supercapacitors, ChemistrySelect, 3 (2018) 8505-8510. DOI: 10.1002/slct.201801174

Bureekaew S., Shimomura S., Kitagawa S., Chemistry and application of flexible porous coordination polymers, Science and Technology of Advanced Materials, 9 (2008) 014108. DOI: 10.1088/1468-6996/9/1/014108

Cao L., Liu Y., Zhang B., Lu L., In situ controllable growth of Prussian blue nanocubes on reduced graphene oxide: facile synthesis and their application as enhanced nanoelectrocatalyst for $\mathrm{H}_{2} \mathrm{O}_{2}$ reduction, ACS Applied Materials \& Interfaces, 2 (2010) 2339-2346. DOI: 10.1021/am100372m

Daneshvar F., Aziz A., Abdelkader A.M., Zhang T., Sue H.-J., Welland M.E., Porous $\mathrm{SnO}_{2}-\mathrm{Cu}_{x} \mathrm{O}$ nanocomposite thin film on carbon nanotubes as electrodes for high performance supercapacitors, Nanotechnology, 30 (2018) 015401. DOI: 10.1088/1361-6528/aae5c6

Doty F.P., Bauer C.A., Skulan A.J., Grant P.G., Allendorf M.D., Scintillating metal-organic frameworks: a new class of radiation detection materials, Advanced Materials, 21 (2009) 95-101. DOI: 10.1002/adma.200801753

Fletcher D.C., Hunter R., Xia W., Smales G.J., Pauw B.R., Blackburn E., Kulak A., Xin H., Schnepp Z., Scalable synthesis of dispersible iron carbide $\left(\mathrm{Fe}_{3} \mathrm{C}\right)$ nanoparticles by 'nanocasting', Journal of Materials Chemistry A, 7 (2019) 19506-19512. DOI: 10.1039/C9TA06876G

Ge C.-X., Li P.-J., Lai J.-H., Qiu P., In situ synthesis and characterization of Prussian blue nanocubes on graphene oxide and its application for $\mathrm{H}_{2} \mathrm{O}_{2}$ reduction, Indian Journal of Chemistry, 57A (2018) 26-33. http://nopr.niscair.res.in/handle/ $123456789 / 43488$
Ishizaki M., Tsuruta S., Kanaizuka K., Sakamoto M., Kawamoto T., Tanaka H., Kurihara M., Growth of Pt subnano clusters on limited surface areas of Prussian blue nanoparticles, Journal of Inorganic and Organometallic Polymers and Materials, 23 (2013) 216-222. DOI: 10.1007/s10904-012-9721-9

Islam M.N., Gorgannezhad L., Masud M.K., Tanaka S., Hossain M.S.A., Yamauchi Y., Nguyen N.-T., Shiddiky M.J.A., Graphene-oxide-loaded superparamagnetic iron oxide nanoparticles for ultrasensitive electrocatalytic detection of microRNA, ChemElectroChem, 5 (2018) 2488-2495. DOI: 10.1002/celc.201800339

Kaneti Y.V., Tang J., Salunkhe R.R., Jiang X., Yu A., Wu K.C.-W., Yamauchi Y., Nanoarchitectured design of porous materials and nanocomposites from metal-organic frameworks, Advanced Materials, 29 (2017) 1604898. DOI: 10.1002/ adma.201604898

Lee T., Min S.H., Gu M., Jung Y.K., Lee W., Lee J.U., Seong D.G., Kim B.-S., Layer-by-layer assembly for graphenebased multilayer nanocomposites: synthesis and applications, Chemistry of Materials, 27 (2015) 3785-3796. DOI: 10.1021/acs.chemmater.5b00491

Lin W., Rieter W.J., Taylor K.M.L., Modular synthesis of functional nanoscale coordination polymers, Angewandte Chemie International Edition, 48 (2009) 650-658. DOI: 10.1002/anie. 200803387

Liu H.D., Zhang J.L., Xu D.D., Huang L.H., Tan S.Z., Mai W.J., Easy one-step hydrothermal synthesis of nitrogen-doped reduced graphene oxide/iron oxide hybrid as efficient supercapacitor material, Journal of Solid State Electrochemistry, 19 (2015) 135-144. DOI: 10.1007/s10008-014-2580-2

Machala L., Zoppellaro G., Tuček J., Šafářová K., Marušák Z., Filip J., Pechoušek J., Zbořil R., Thermal decomposition of Prussian blue microcrystals and nanocrystals - iron(iii) oxide polymorphism control through reactant particle size, RSC Advances, 3 (2013) 19591-19599. DOI: 10.1039/ C3RA42233J

Paolella A., Faure C., Timoshevskii V., Marras S., Bertoni G., Guerfi A., Vijh A., Armand M., Zaghib K., A review on hexacyanoferrate-based materials for energy storage and smart windows: challenges and perspectives, Journal of Materials Chemistry A, 5 (2017) 18919-18932. DOI: 10.1039/C7TA05121B

Park S., An J., Potts J.R., Velamakanni A., Murali S., Ruoff R.S., Hydrazine-reduction of graphite- and graphene oxide, Carbon, 49 (2011) 3019-3023. DOI: 10.1016/j. carbon.2011.02.071

Ren S., Li R., Meng X., Li H., Self-assembly of reduced graphene oxide at liquid-air interface for organic field-effect transistors, Journal of Materials Chemistry, 22 (2012) 6171-6175. DOI: $10.1039 / \mathrm{C} 2 J M 16232 \mathrm{~F}$

Roy X., Hui J.K.-H., Rabnawaz M., Liu G., MacLachlan M.J., Soluble Prussian blue nanoworms from the assembly of metal-organic block ionomers, Angewandte Chemie International Edition, 50 (2011) 1597-1602. DOI: 10.1002/ anie. 201005537

Salunkhe R.R., Kaneti Y.V., Kim J., Kim J.H., Yamauchi Y., Nanoarchitectures for metal-organic framework-derived nanoporous carbons toward supercapacitor applications, 
Ackounts of Chemical Research, 49 (2016) 2796-2806. DOI:

NO.1021/acs.accounts.6b00460

Salunkhe R.R., Tang J., Kamachi Y., Nakato T., Kim J.H., Yamauchi Y., Asymmetric supercapacitors using 3D nanoporous carbon and cobalt oxide electrodes synthesized from a single metal-organic framework, ACS Nano, 9 (2015) 6288-6296. DOI: $10.1021 /$ acsnano.5b01790

Sun D., Wang H., Deng B., Zhang H., Wang L., Wan Q., Yan X., Qu M., A Mn-Fe based Prussian blue Analogue@Reduced graphene oxide composite as high capacity and superior rate capability anode for lithium-ion batteries, Carbon, 143 (2019) 706-713. DOI: 10.1016/j.carbon.2018.11.078

Tanaka S., Salunkhe R.R., Kaneti Y.V., Malgras V., Alshehri S.M., Ahamad T., Zakaria M.B., Dou S.X., Yamauchi Y., Hossain M.S.A., Prussian blue derived iron oxide nanoparticles wrapped in graphene oxide sheets for electrochemical supercapacitors, RSC Advances, 7 (2017) 33994-33999. DOI: 10.1039/C7RA03179C

Vermisoglou E.C., Devlin E., Giannakopoulou T., Romanos G., Boukos N., Psycharis V., Lei C., Lekakou C., Petridis D., Trapalis C., Reduced graphene oxide/iron carbide nanocomposites for magnetic and supercapacitor applications, Journal of Alloys and Compounds, 590 (2014) 102-109. DOI: 10.1016/j.jallcom.2013.11.087

Wang Z.-L., Sun K., Henzie J., Hao X., Ide Y., Takei T., Bando Y., Yamauchi Y., Electrochemically in situ controllable assembly of hierarchically-ordered and integrated inorganic-carbon hybrids for efficient hydrogen evolution, Materials Horizons, 5 (2018) 1194-1203. DOI: 10.1039/C8MH00773J

Zakaria M.B., Nanostructuring of nanoporous iron carbide spheres via thermal degradation of triple-shelled Prussian blue hollow spheres for oxygen reduction reaction, $\mathrm{RSC}$ Advances, 6 (2016) 10341-10351. DOI: 10.1039/C5RA24357B

Zakaria M.B., Tan H., Kim J., Badjah A.Y., Naushad M., Habila M., Wabaidur S., Alothman Z.A., Yamauchi Y., Lin J., Structurally controlled layered $\mathrm{Ni}_{3} \mathrm{C}$ /graphene hybrids using cyano-bridged coordination polymers, Electrochemistry Communications, 100 (2019) 74-80. DOI: 10.1016/j.elecom. 2019.01.004

Zhu K., Chen C., Xu M., Chen K., Tan X., Wakeel M., Alharbi N.S., In situ carbothermal reduction synthesis of Fe nanocrystals embedded into $\mathrm{N}$-doped carbon nanospheres for highly efficient U(VI) adsorption and reduction, Chemical Engineering Journal, 331 (2018) 395-405. DOI: 10.1016/j. cej.2017.08.126

\section{Authors' Short Biographies}
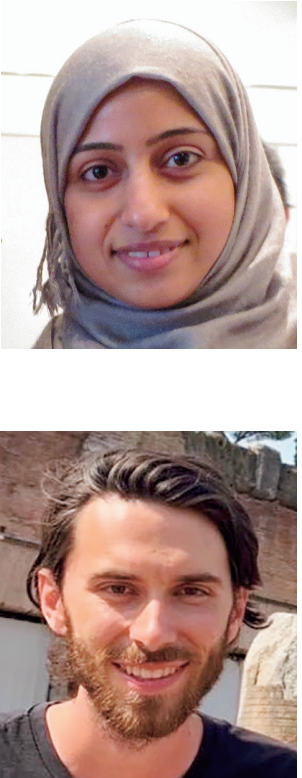

\section{Alowasheeir Azhar}

Dr. Alowasheeir Azhar received her BSc in Energy Engineering (2013), MSc in Applied Science and Chemistry (2015) from Tokai University (Japan), and Ph.D. (2019) in Department of Nanoscience and Nanoengineering at Waseda University (Japan). She received the Custodian of The Two Holy Mosques' overseas scholarship program (2007 to 2019) from Kingdom of Saudi Arabia. Her current research interest is the design for hybrid materials and nanoporous materials.

\section{Jacob Earnshaw}

Jacob Earnshaw received his BSc (Chemistry) degree from the School of Chemistry and Molecular Biosciences at the University of Queensland, Australia in 2018. Currently, he is an Honours student under the supervision of Professor Yusuke Yamauchi, and his research pursues the synthesis of nanoarchitectured materials for energy storage and electrocatalysis. 


\section{Authors' Short Biographies}

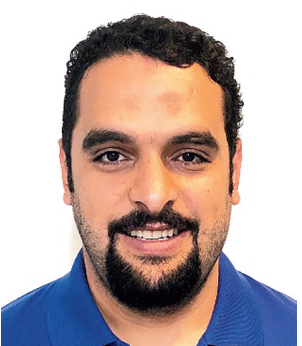

\section{Mohamed B. Zakaria}

Dr. Mohamed B. Zakaria is a senior researcher at Australian Institute for Bioengineering and Nanotechnology (AIBN), the University of Queensland, Australia and a Lecturer of Physical Chemistry, Department of Chemistry, Faculty of Science, Tanta University, Egypt. He received his BSc in Chemistry and MSc in Physical Chemistry from Tanta University. Then, he received his $\mathrm{PhD}$ in Engineering in 2016 from Waseda University, Japan. He did his postdoctoral research at NIMS-MANA, Tsukuba, Japan as a JSPS Fellow from 2016-2018. His area of interest is in the fields of Chemistry, Materials Design, Hybrid Materials, Energy, Catalysis, Dielectric Capacitors, Ferroelectric Materials, and Applied Nanomaterials.

\section{Ping Cheng}

Ping Cheng received a MSc in biotechnology in 2019 at the University of Queensland. He is currently studying for Ph.D. in the group of Prof. Yusuke Yamauchi, Australian Institute for Bioengineering and Nanotechnology. His research interests include environmental applications and biological applications of porous materials.

\section{Yusuf Valentino Kaneti}

Dr. Yusuf Valentino Kaneti received his PhD from the University of New South Wales in July 2014 under the supervision of Prof. Aibing Yu. He joined the group of Prof. Yusuke Yamauchi at the National Institute of Materials Science as a JSPS Postdoctoral Fellow in September 2016. He is now working as a MANA Research Associate at NIMS. His research interest is focused on the fabrication of functional porous materials for energy and environmental applications.

\section{Md. Shahriar A. Hossain}

Dr. Md Shahriar A. Hossain is currently a Senior Lecturer in the School of Mechanical and Mining Engineering at UQ. He obtained his Ph.D degree in Materials Science and Engineering, The University of Wollongong (UoW), Australia in 2008. He was then employed as a Postdoctoral Research Fellow at the University of Geneva, Switzerland. He was awarded the prestigious DECRA Fellowship from the Australian Research Council (ARC) in 2013. His research experience in various institutes and in industry around the world has been mainly focused on fabrication and characterization of superconducting materials and magnetically triggered nanoparticles for medical and energy applications. 


\section{Authors' Short Biographies}

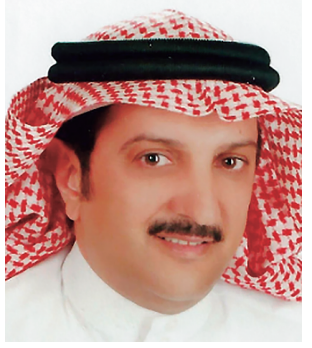

\section{Saad M. Alshehri}

Prof. Saad M Alshehri received his Ph.D. in Inorganic Chemistry from the Leicester University, England in 1992. He worked at Northeastern University, Boston and the Leicester University as a post-doctoral fellow. Prior to join to King Saud University, Prof. Saad was an Assistant Professor of Chemistry at King Khalid Military Academy, Riyadh, Saudi Arabia. His research interests are in synthesis of inorganic materials and their industrial applications. He has synthesized heat resistant and thermally stable material. He has also investigated inorganic and hybrid materials for electro-catalyst and for energy storage devices such as electrodes for lithium ions batteries and supercapacitors.

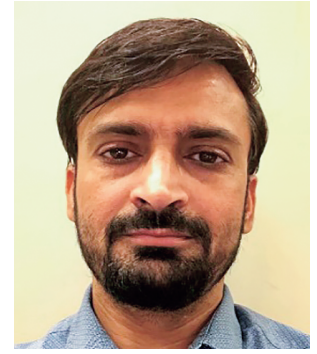

\section{Tansir Ahamad}

Dr. Tansir Ahamad received his PhD degree in 2006 from Jamia Millia Islamia, New Delhi (INDIA). After his graduation, he was employed as a post-doc at the University of Western cape (South Africa). At the conclusion of his post-doc position he joined King Saud University, Saudi Arab in 2008 and was employed as an Assistant Professor. From March 2020, he has started his academic employment as a full professor in the department of Chemistry at King Saud University. His major research interest is in the fabrication of porous nanomaterials, organic-inorganic hybrid materials, and their applications in energy and environments.

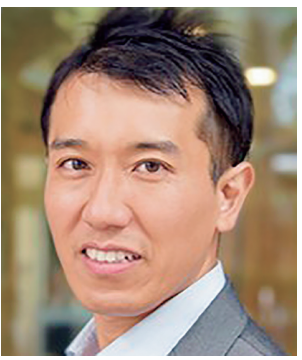

\section{Yusuke Yamauchi}

Prof. Yusuke Yamauchi received his bachelor's degree (2003), master's degree (2004), and $\mathrm{PhD}$ degree (2007) from Waseda University, Japan. After that, he joined the National Institute for Materials Science to start his research group. Currently, he is a full professor at the School of Chemical Engineering and a senior group leader at AIBN in The University of Queensland. He concurrently serves as an associate editor of J Mater Chem A (RSC) and Chem Eng J (Elsevier). He has published over 750 papers with $>36,000$ citations (h-index 97, Web of Science ${ }^{\circledR}$ ). He was selected as the Highly Cited Researchers in Chemistry in 2016-2019.

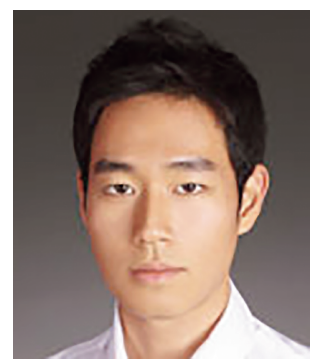

\section{Jongbeom Na}

Dr. Jongbeom Na received his Ph.D. degree (2017) from the Department of Chemical and Biomolecular Engineering at Yonsei University (Republic of Korea). Until 2018, he worked as a Research Engineer at Chemical Laboratory of SK Chemicals Co., Ltd. Currently, he is working as a research fellow at AIBN, the University of Queensland and MANA, NIMS. His major research interest is in the design and synthesis of functional nanomaterials, organic-inorganic hybrid materials, and their applications. 